ARTICLE

\title{
Acoustic-optical phonon up-conversion and hot-phonon bottleneck in lead-halide perovskites
}

Jianfeng Yang${ }^{1}$, Xiaoming Wen¹,2, Hongze Xia¹, Rui Sheng${ }^{1}$, Qingshan Ma1, Jincheol Kim¹, Patrick Tapping ${ }^{3}$, Takaaki Harada ${ }^{3}$, Tak W. Kee ${ }^{3}$, Fuzhi Huang ${ }^{4}$, Yi-Bing Cheng ${ }^{5}$, Martin Green ${ }^{1}$, Anita Ho-Baillie', Shujuan Huang ${ }^{1}$, Santosh Shrestha', Robert Patterson ${ }^{1} \&$ Gavin Conibeer ${ }^{1}$

The hot-phonon bottleneck effect in lead-halide perovskites $\left(\mathrm{APbX}_{3}\right)$ prolongs the cooling period of hot charge carriers, an effect that could be used in the next-generation photovoltaics devices. Using ultrafast optical characterization and first-principle calculations, four kinds of lead-halide perovskites $\left(\mathrm{A}=\mathrm{FA}^{+} / \mathrm{MA}^{+} / \mathrm{Cs}^{+}, \mathrm{X}=\mathrm{I}^{-} / \mathrm{Br}^{-}\right)$are compared in this study to reveal the carrier-phonon dynamics within. Here we show a stronger phonon bottleneck effect in hybrid perovskites than in their inorganic counterparts. Compared with the caesium-based system, a 10 times slower carrier-phonon relaxation rate is observed in $\mathrm{FAPb}_{3}$. The up-conversion of low-energy phonons is proposed to be responsible for the bottleneck effect. The presence of organic cations introduces overlapping phonon branches and facilitates the up-transition of low-energy modes. The blocking of phonon propagation associated with an ultralow thermal conductivity of the material also increases the overall up-conversion efficiency. This result also suggests a new and general method for achieving long-lived hot carriers in materials.

\footnotetext{
${ }^{1}$ Australian Centre for Advanced Photovoltaics, School of Photovoltaics and Renewable Energy Engineering, University of New South Wales, Sydney, New South Wales 2052, Australia. ${ }^{2}$ Centre for Micro-Photonics, Swinburne University of Technology, Melbourne, Victoria 3122, Australia. ${ }^{3}$ Department of Chemistry, The University of Adelaide, Adelaide, South Australia 5005, Australia. ${ }^{4}$ State Key Lab of Advanced Technologies for Materials Synthesis and Processing, Wuhan University of Technology, Wuhan 430070, China. ${ }^{5}$ Department of Materials Science and Engineering, Monash University, Melbourne, Victoria 3800, Australia. Correspondence and requests for materials should be addressed to X.W. (email: xm_wen@hotmail.com).
} 
S ignificant progress has been achieved in metal-halide perovskite $\left(\mathrm{APbX}_{3}\right)$ solar cells with the certified efficiency record now exceeding $20 \%$, just a few years after the first solid-state device was reported to have an efficiency of $9.7 \%$ in 2012 (refs 1-5). The photophysics and carrier dynamics in lead-halide perovskites have been intensively studied in the last few years because of their promising performance in the conventional photovoltaic devices. The rapid emergence of lead-halide perovskites is attributed to their outstanding optoelectronic properties, including superb optical absorption, high ambipolar charge mobility and appropriate band gap ${ }^{6,7}$. Recently, a significant hot-phonon bottleneck effect in carrier thermalization was also observed in lead-halide perovskites ${ }^{8,9}$, which indicates potential applications of these kinds of materials in the advanced concept of hot carrier optoelectronics.

The so-called hot-phonon bottleneck effect denotes a phenomenon where the relaxation rate of a non-equilibrium carrier-phonon system is reduced when the carrier injection level is high ${ }^{10-14}$. Cooling of photo-generated hot carriers in a material dissipates the absorbed optical energy as lattice heat via longitudinal optical (LO) phonon emission and decay. Thermalization by this mechanism leads to about $50 \%$ of the energy losses in a traditional single junction solar cell ${ }^{15}$. A strong phonon bottleneck effect is helpful to establish a long-lived hot carrier population, which is critical to achieve a working hot carrier photovoltaic device ${ }^{16-18}$ and break the Shockley-Queisser limit ${ }^{19}$ for photovoltaic energy conversion. Hot carrier properties of materials are also important in photodetection ${ }^{20}$, photocatalysis $^{21}$ and light emission ${ }^{22,23}$ to improve the power efficiency and carrier dynamics, especially in high-power optoelectronic applications such as lasing ${ }^{24-26}$.

Although several mechanisms have been suggested for the hot carrier dynamics on sub-picosecond timescales in lead-halide perovskites $^{27-29}$, a comprehensive understanding of the phonon bottleneck effect occurring on longer timescales (up to thousand picoseconds) is still lacking. Slow carrier cooling and the bottleneck phenomenon can be caused by different mechanisms, such as a modified carrier-phonon interaction and a blocked hot-phonon decay in general. However, no clear conclusion has been made in previous studies for lead-halide perovskites.

In this study, we attempt to provide further insight on the phonon bottleneck effect in the lead-halide perovskite family. We compare the hot carrier and phononic properties among four typical kinds of lead-halide perovskites used in photovoltaics, including $\mathrm{CH}_{3} \mathrm{NH}_{3} \mathrm{PbI}_{3}\left(\mathrm{MAPbI}_{3}\right), \mathrm{HC}\left(\mathrm{NH}_{2}\right)_{2} \mathrm{PbI}_{3} \quad\left(\mathrm{FAPbI}_{3}\right)$, $\mathrm{CH}_{3} \mathrm{NH}_{3} \mathrm{PbBr}_{3}\left(\mathrm{MAPbBr}_{3}\right)$ and $\mathrm{CsPbIBr}{ }_{2}$. By varying the A-site cations $\left(\mathrm{MA}^{+}, \mathrm{FA}^{+}, \mathrm{Cs}^{+}\right)$and $\mathrm{X}$-site halide anions $\left(\mathrm{I}^{-}, \mathrm{Br}^{-}\right)$, we try to elucidate the impact of lattice composition on the carrier cooling properties. A complete picture of hot carrier relaxation in lead-halide perovskites is presented using the ultrafast transient absorption (TA) technique. Further studies of the phonon band structures and emission rates reveal the dynamics behind the relaxation. The phonon bottleneck effect in these materials is explained by acoustic phonon up-conversion, which leads to a LO phonon emission rate that is 10 times slower in $\mathrm{FAPbI}_{3}$ compared with the inorganic counterpart under the same experimental conditions. The presence of rotatable groups on the organic sub-lattice is proposed to be the essential reason for this efficient up-transition dynamics. To the best of our knowledge, this mechanism has been ignored previously in lead-halide perovskite research and is also rarely discussed by the hot carrier research community. This study connects a variety of material properties in lead-halide perovskites together. The proposed mechanism is also enlightening for the future exploration of new functional hot carrier materials.

\section{Results}

Power-dependent carrier temperature decay. All the samples used in this study, including $\mathrm{MAPbI}_{3}, \mathrm{FAPbI}_{3}, \mathrm{MAPbBr}_{3}$ and $\mathrm{CsPbIBr}$, were prepared on glass substrates with a thickness about $300 \mathrm{~nm}$. The sample preparations are described in the Methods section and the detailed characterizations have been reported elsewhere ${ }^{30-33}$. The fundamental absorption band gaps were determined to be $1.69,1.55,2.31$ and $2.05 \mathrm{eV}$ for the $\mathrm{MAPbI}_{3}, \mathrm{FAPbI}_{3}, \mathrm{MAPbBr}_{3}$ and $\mathrm{CsPbIBr}$ samples, respectively. On the basis of the fabrication methods, all samples are optimized in conventional photovoltaic applications, which are also sufficiently stable during the measurements to ensure that accurate and reproducible experimental data are obtained. All the femtosecond pump-probe TA measurements (see 'Methods' section) were conducted under identical ambient conditions at room temperature $(295 \mathrm{~K})$. A pump pulse with a wavelength of $400 \mathrm{~nm}$ and duration of $100 \mathrm{fs}$ was used to excite carriers well above the band gap for all the samples, while an ultrashort broadband super-continuum laser covering the whole visible range was used as the probe. The pump-induced change in the
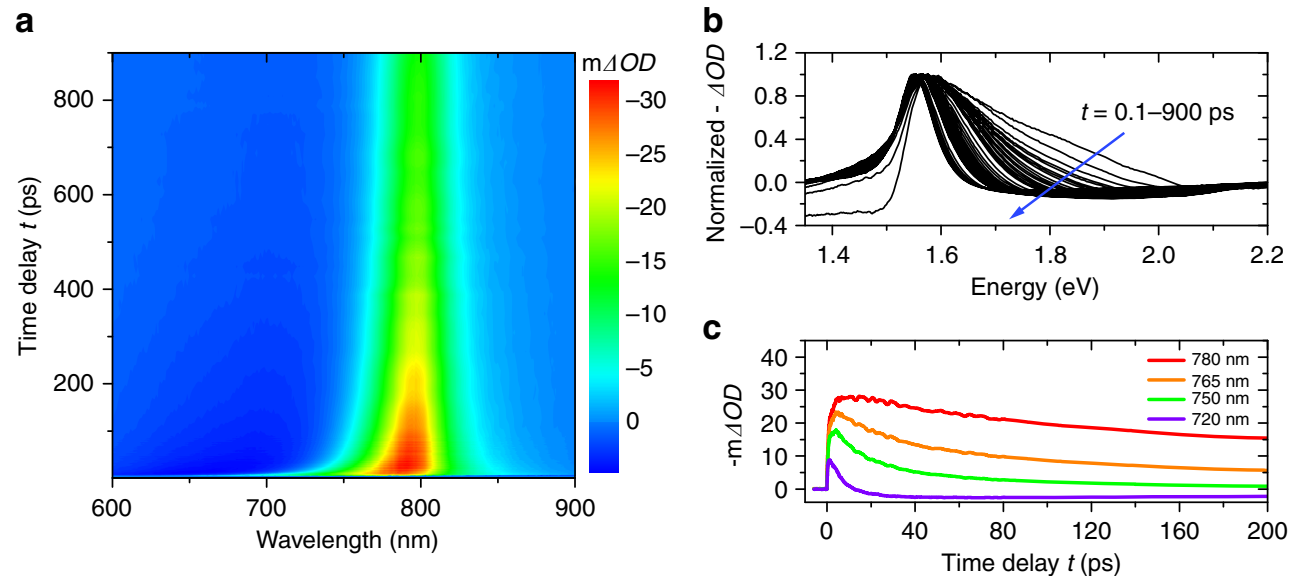

Figure 1 | Transient absorption spectra of the $\mathbf{F A P b}_{\mathbf{3}}$ sample. (a) 3D TA spectrum pumped at $400 \mathrm{~nm}$ with an initial carrier concentration $N_{0}=4.84 \times 10^{18} \mathrm{~cm}^{-3}$. The initial broadening of the bleaching signal extending above the apparent band gap energy indicates the temporary presence of hot carriers. (b) Normalized negative TA spectra $(-\Delta O D)$ at different pump-probe time delays $t$ from 0.1 to about 900 ps. (c) Decay of the bleaching signal given as minus milli $\triangle O D(-\mathrm{m} \triangle O D)$ at different wavelengths showing carrier relaxation kinetics. 
absorption spectrum was acquired as a function of time delay. The carrier relaxation dynamics were then resolved via the bleaching in the spectra above the band edge under different excitation fluences.

Figure 1 shows the TA spectrum obtained from the $\mathrm{FAPbI}_{3}$ sample under a pump fluence of $90 \mu \mathrm{Jcm}^{-2}$ with an initial carrier concentration around $N_{0}=4.84 \times 10^{18} \mathrm{~cm}^{-3}$ (see Supplementary Methods for carrier injection estimation). Sustained absorption bleaching is peaked around the apparent band gap (about $1.55 \mathrm{eV}$ ) and broadening into the high-energy region, whose gradual narrowing as shown in Fig. 1b,c exhibits the carrier thermalization process. The so-called phonon bottleneck effect is intuitively demonstrated by a power-dependent measurement. The bleaching intensity in the high-energy region is markedly reduced under a pump fluence of $10 \mu \mathrm{Jm}^{-2}$, indicating a variation in carrier relaxation dynamics under different concentrations (Supplementary Fig. 1). Under similar experimental conditions, power-dependent TA measurements were also performed on the other three samples. The measured 3D TA spectra under the maximum pump fluence (about $100 \mu \mathrm{J} \mathrm{cm}^{-2}$ ) in each sample are given in Supplementary Fig. 2.

The carrier temperature $T_{c}$ is obtained by fitting the highenergy tail of the bleaching spectrum above the band edge ${ }^{34-36}$ whose line shape is approximated to be a modified MaxwellBoltzmann distribution (see Supplementary Methods for carrier temperature fitting section 1). The fittings are then conducted among all the measured TA spectra following the same tail selection scheme with a careful sensitivity analysis to ensure reliable and comparable fitting results. The fitted carrier temperatures as a function of time delay $t$ are summarized in Fig. 2 (see Supplementary Methods section 2 and section 3 for carrier temperature fitting).

Among different lead-perovskite samples, two primary features are shared according to their cooling curves. First, all the samples

a

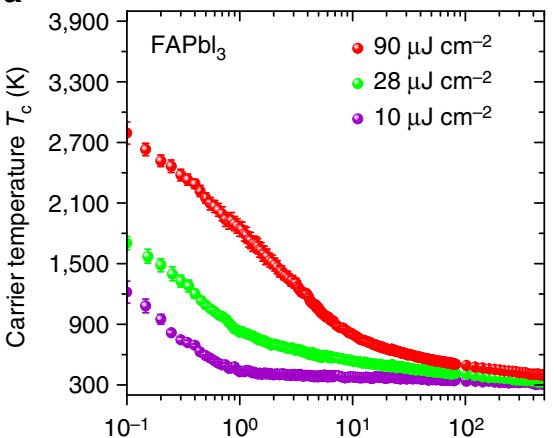

C

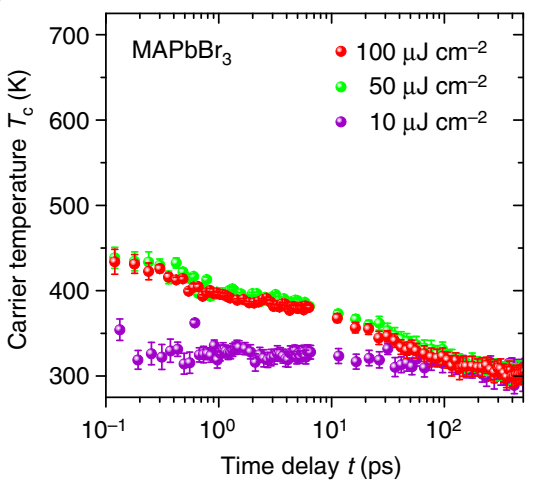

show an evident temperature-dependent carrier cooling rate, which is apparently decreased when the carrier temperature is lower. Although the primary carrier temperature decrease roughly occurs within the first $10 \mathrm{ps}$ in these samples, a subsequent slower cooling rate extends the overall relaxation period up to several hundred picoseconds. On the same cooling curve of $\mathrm{FAPbI}_{3}$ under $90 \mu \mathrm{J} \mathrm{cm}{ }^{-2}$ fluence in Fig. 2a, an average cooling lifetime of 25 and 124 ps are observed beginning at $T_{\mathrm{c}}=1,500 \mathrm{~K}$ and $700 \mathrm{~K}$, respectively. These average lifetimes are obtained using a double-exponential fitting, which is applied from the selected initial high carrier temperatures to the end of the cooling curve.

Second, two stages of the carrier cooling process are identified from Fig. 2, whose cooling rates exhibit a different power dependence. The first cooling stage shows a very similar subpicosecond lifetime under different excitation intensities. This stage is dominated by the intrinsic Fröhlich phonon emission corresponding to the typical timescales studied before in leadhalide perovskites ${ }^{37}$. A limited thermalization rate for hot holes is observed in this stage compared with conventional organic semiconductors. A reduced electronic density of state (DOS) near the fundamental valence band maximum was used to explain the restricted relaxation of carriers, which leads to a nearly power-independent cooling rate observed here. Since the electronic band structure near the band edge is primarily dominated by the lead-halide bond ${ }^{37,38}$, similar cooling lifetimes and their power independence are also observed in the inorganic $\mathrm{CsPbIBr}$ sample on this stage. The second cooling stage is then observed in the later period under a sufficiently high fluence (larger than $10 \mu \mathrm{Jm}^{-2}$ here). The cooling lifetime increases with a larger carrier density in the second stage evidently, corresponding to the effect of a phonon bottleneck. Just following the similar double-exponential fitting used above from a fixed initial temperature $T_{\mathrm{c}}=700 \mathrm{~K}$, the cooling lifetime of

b

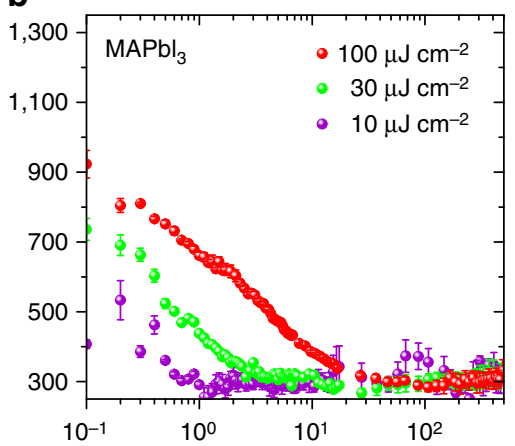

d

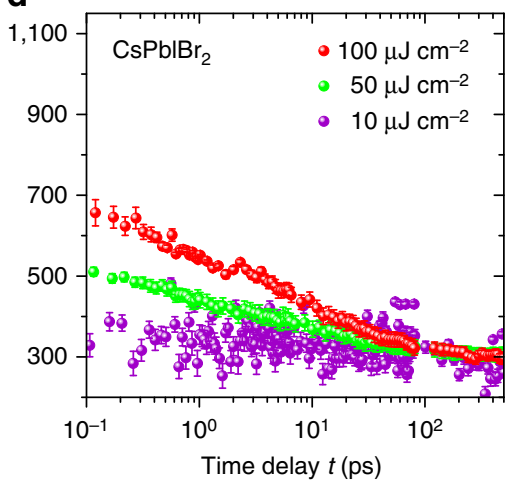

Figure 2 | Time-dependent carrier temperature under different incident fluence. In (a) $\mathrm{FAPbl}_{3}$ (b) $\mathrm{MAPbl}_{3},(\mathbf{c}) \mathrm{MAPbBr}_{3}$ (d) $\mathrm{CsPblBr}{ }_{2}$. The carrier temperatures are extracted by fitting the high-energy tail of the bleaching in the TA spectra. The error bar shows the standard error of the average (s.e.m.) fitting results. 
$\mathrm{FAPbI}_{3}$ grows from 45 to $124 \mathrm{ps}$ when the fluence is tuned from 28 to $90 \mu \mathrm{J} \mathrm{cm}^{-2}$ correspondingly.

Compared with $\mathrm{FAPbI}_{3}$, an average cooling lifetime of only $6 \mathrm{ps}$ beginning from $T_{\mathrm{c}}=700 \mathrm{~K}$ is observed in our $\mathrm{MAPbI}_{3}$ sample under the maximum pumping with a much lower initial carrier temperature. This observation is inconsistent with the previous theoretical and experimental conclusion on $\mathrm{MAPbI}_{3}$, although a power-dependent bottleneck effect is also observed in its second cooling stage. The changes in the organic cation $\left(\mathrm{FA}^{+}\right.$and $\left.\mathrm{MA}^{+}\right)$with the same kind of lead-halide framework should not strongly perturb both the electronic and phononic properties of the material ${ }^{8,27,37,38}$. This significant difference is believed to be caused by the lattice defects. Our previous observation confirms that the $\mathrm{MAPbI}_{3}$ sample prepared by the gas-assisted method (see 'Methods' section) has a large density of sub-band-gap trapping states ${ }^{39}$. The fast carrier relaxation observed here convinces us that a large number of above-bandgap defects are also presented in this sample, which could provide more relaxation pathways for the hot carriers ${ }^{40}$. It is noted that the solar cell made of such a material can still achieve a fairly good energy conversion efficiency up to $17 \%$ (ref. 30). This suggests the relaxation of hot carriers is more sensitive to the presence of lattice defeats, so special attention to material quality is required.

Although the initial carrier temperatures in $\mathrm{MAPbBr}_{3}$ and $\mathrm{CsPbIBr}{ }_{2}$ are lower than that in $\mathrm{FAPbI}_{3}$ and $\mathrm{MAPbI}_{3}$ due to larger band gaps, power-dependent cooling lifetimes are still observed in their second cooling stage. Under a similar carrier concentration, the bottleneck effect between all the materials can be compared by investigating the second cooling stage in a similar carrier temperature range. To intuitively show the difference in the bottleneck effect among these samples, we first compare their average cooling lifetimes fitted below the same carrier temperature $T_{\mathrm{c}}=400 \mathrm{~K}$ for an intermediate fluence $\left(28 \mu \mathrm{J} \mathrm{cm}{ }^{-2}\right.$ for $\mathrm{FAPbI}_{3}, 50 \mu \mathrm{J} \mathrm{cm}{ }^{-2}$ for $\mathrm{MAPbBr}_{3}$ and $\mathrm{CsPbIBr}_{2}$ ). All the samples are then in the second cooling stage with a similar carrier density of about $N_{0}=2 \times 10^{18} \mathrm{~cm}^{-3}$ for ready comparison. A cooling lifetime of about $305 \mathrm{ps}$ is observed in the $\mathrm{FAPbI}_{3}$ sample in this period, while only a lifetime of 71 and $37 \mathrm{ps}$ are observed in the $\mathrm{MAPbBr}_{3}$ and $\mathrm{CsPbIBr}_{2}$ sample, respectively. When the excitation intensity increases to the maximum one used in our measurements with a carrier density of about $N_{0}=5 \times 10^{18} \mathrm{~cm}^{-3}$, the cooling lifetime of $\mathrm{FAPbI}_{3}$ is significantly increased to around $1 \mathrm{~ns}$ after $T_{\mathrm{c}}=400 \mathrm{~K}$ while only about 48 ps is achieved in $\mathrm{CsPbIBr}_{2}$.

An unusual saturation effect was observed in the $\mathrm{MAPbBr}_{3}$ sample (Fig. 2c), where both the TA spectra and carrier temperature show nearly the same result when the excitation is larger than $50 \mu \mathrm{Jcm}^{-2}$, corresponding to a carrier density about $N_{0}=2.69 \times 10^{18} \mathrm{~cm}^{-3}$. Since there is no evidence of either saturation in absorption at the pumping wavelength $(400 \mathrm{~nm})$ or a lasing effect in $\mathrm{MAPbBr}_{3}$ according to the TA spectrum, we consider this observation to be a result of Auger-Impact Ionization (AI) processes $^{41}$. It has been reported that the Auger coefficient in $\mathrm{MAPbBr}_{3}$ is about four times larger than that in $\mathrm{MAPbI}_{3}$ (ref. 42). Triggered by a sufficiently high excess carrier concentration, it is possible that a strong multi-particle interaction occurs in this material, limiting the change of carrier temperature under a higher excitation power density.

Phonon relaxation and bottleneck effect. To understand the mechanism behind the bottleneck effect observed in lead-halide perovskites, we first review some general understandings of carrier-phonon dynamics in solid-state polar semiconductors. The carrier thermalization is accompanied by the emission of LO phonons, which then decay to the low-energy acoustical modes and lead to local lattice heating. With an effective thermal transfer to the surroundings via acoustic phonon propagation, the carrier's excess kinetic energy is eventually dissipated in an irreversible manner. Therefore, three distinct relaxation stages exist: (1) carrier-phonon scattering (Fröhlich interaction); (2) optical phonon decay to acoustic phonons; (3) acoustic phonon propagation to the far-field region in the material (thermal conduction). Correspondingly, in a non-quantized system with continuous electronic DOS, possible blocking mechanisms can be introduced in each relaxation stage to prolong the overall cooling period of hot carriers: (1) thermal isolation between the optical phonon and carrier population; (2) reducing the phononic DOS; (3) acoustic phonon up-conversion to optical phonon.

Strong thermal isolation required by the first mechanism is very difficult to achieve due to the spontaneous interaction between the carriers and LO phonons. In lead-halide perovskites, the Fröhlich interaction mainly occurs between the charge carriers and lead-halide bond 27,37 , which governs the carrier relaxation in the first cooling stage. Although the large polaron effect proposed recently ${ }^{29}$ demonstrates that a reduced carrierphonon coupling may exist in the organic-inorganic lead-halide perovskites, a Fröhlich scattering rate similar to the typical III-V semiconductor materials, such as GaAs, has also been reported $^{8,27}$. In particular, since the electron-phonon coupling occurs constantly during carrier thermalization, just a change of Fröhlich coupling also cannot explain why a power-dependent carrier cooling rate is only observed in the second cooling stage while the rate in the first stage remains nearly unaffected. Therefore, it can be ruled out as a major cause of the phonon bottleneck effect here. The second method is one of the major mechanisms utilized in the current carrier thermalization engineering. In typical semiconductor systems, the decay mechanisms of LO phonon to low-energy acoustic modes include the symmetric Klemens-decay and anti-symmetric Ridley-decay ${ }^{17}$. Opening the phonon band gap (between the optical and acoustical branches) using compounds with a large atomic mass-difference has been intensively studied before ${ }^{17,18,43,44}$ to slow carrier cooling, since the Klemens-decay mechanism is blocked if the phonon band gap is greater than twice of the highest acoustic phonon energy. The third blocking mechanism happens in the last stage of carrier cooling where the induced local lattice heat finally dissipates to the surroundings. An efficient acoustic phonon up-conversion can recycle the thermal energy back and reheat carriers ${ }^{10}$.

With this background knowledge in mind, we first compare the phonon band structures of these materials. The phonon band structures of $\mathrm{FAPbI}_{3}, \mathrm{MAPbI}_{3}, \mathrm{MAPbBr}_{3}$ and $\mathrm{Cs} \mathrm{PbBr}_{3}$ (as an analogue of $\mathrm{CsPbIBr}$ ) were calculated from first-principles using density functional theory (see 'Methods' section). All the structures were optimized based on a pseudocubic lattice, which has been extensively used to predict properties for these materials ${ }^{45-47}$. The calculation results have also been compared with previous reports to ensure a good estimation of the phonon energy distribution. Figure 3 shows the calculated phonon band structures (below $200 \mathrm{~cm}^{-1}$ ). All the acoustic bands are plotted as red curves. The imaginary phonon modes with a negative frequency result from the metastable lattice of the perovskite where the permanent displacement of the atoms is possible through distortions of the crystal lattice ${ }^{37,48}$. The projected density of states on each atom is also given in Fig. 3 to show the detailed contributions from each sub-lattice.

Evidently, no complete phonon band gap is observed in these four kinds of perovskite, therefore, there is no significant blocking effect from the second mechanism mentioned above. Compared 

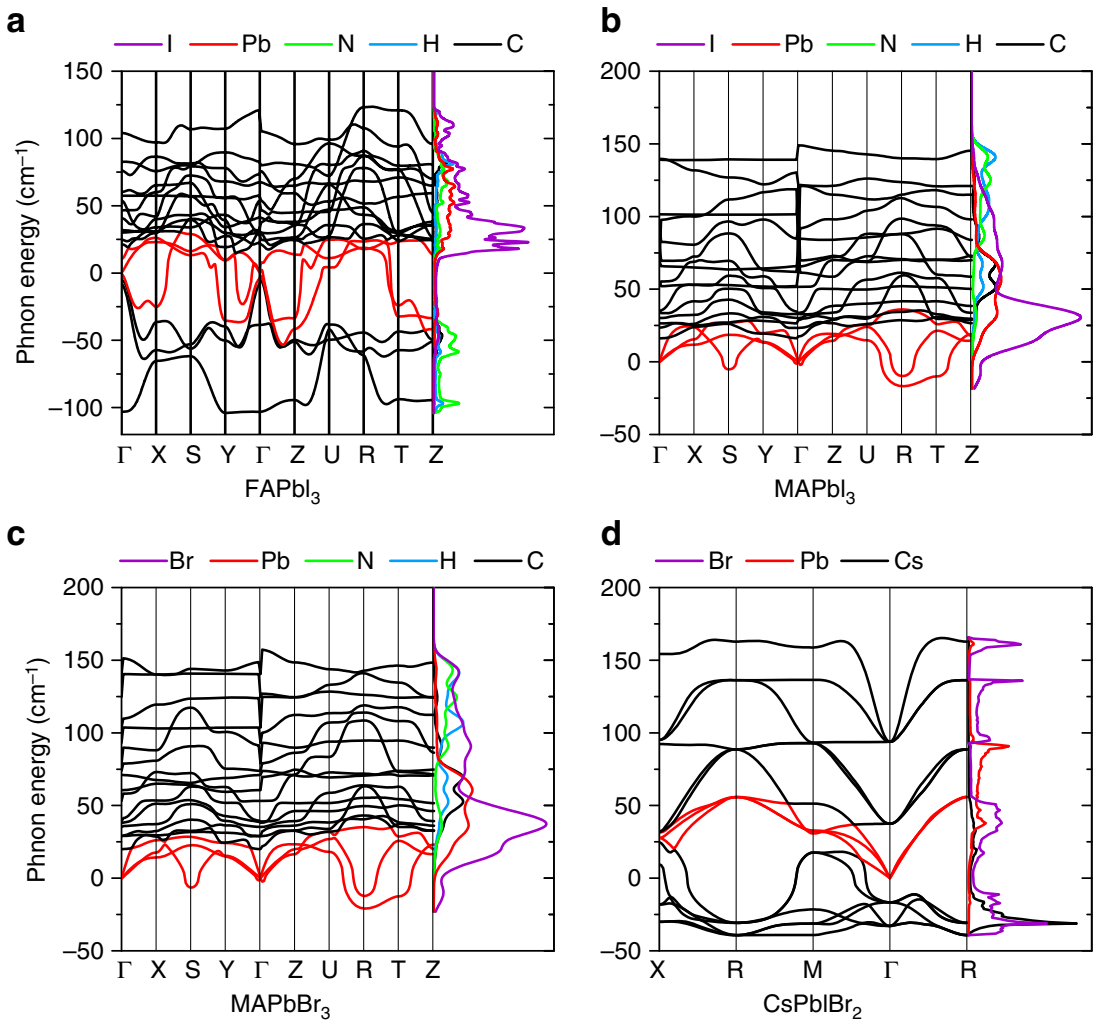

Figure 3 | Phonon band structure and projected phonon DOS. In (a) $\mathrm{FAPbl}_{3} ;(\mathbf{b}) \mathrm{MAPbl}_{3} ;$ (c) $\mathrm{MAPbBr}_{3} ;$ (d) $\mathrm{CsPbBr}_{3}$. The projected phonon DOS are given in arbitrary units (a.u.) and show the contributions of the organic cation and inorganic sub-lattice respectively. The acoustic bands are plotted in red.

with the inorganic type $\mathrm{Cs} \mathrm{PbBr}_{3}$, lattice vibration properties in the hybrid types are strongly altered by the presence of organic cations according to Fig. 3. Different to $\mathrm{Cs}^{+}$, whose vibrational modes are primarily distributed over the acoustic branches in $\mathrm{CsPbBr}$, the phonon modes relating to the organic cations show an optical-like character with a good overlap to the lead-halide optical modes in the range roughly from 30 to $100 \mathrm{~cm}^{-1}$. This result is consistent with the previous Raman observations ${ }^{49}$ on $\mathrm{MAPbI}_{3}$ that the libration and torsion of the $\mathrm{MA}^{+}$is coupled with the lead/halide ions and lead to a series of low-energy optical modes in the range of $60-100 \mathrm{~cm}^{-1}$. This unique phonon structure implies that although the organic cations do not govern the Fröhlich interaction directly, the overlapping vibration modes can still allow them to participate in the carrier-phonon relaxation through phonon-phonon scattering.

With the influence of non-propagating vibrations on organic cation, most of the optical phonon modes below $200 \mathrm{~cm}^{-1}$ in the hybrid types show a relatively flat band dispersion across the first Brillouin zone compared with the inorganic case. A cascade decay of LO phonons may be supported by this mini-band-like structure to slow phonon relaxation. However, it cannot fully explain the much slower carrier cooling lifetime of $\mathrm{FAPbI}_{3}$ in the second stage (below $T_{\mathrm{c}}=400 \mathrm{~K}$ ) observed above comparing with $\mathrm{MAPbBr}_{3}$, although a similar mini-band-like optical branches are shown in both of them. A series of high-energy (larger than $200 \mathrm{~cm}^{-1}$ ) quasi-static modes are also observed in the hybrid perovskites, which result from the resonant vibrations of $\mathrm{H}$ atoms primarily (Supplementary Fig. 3). However, these high-energy modes are considered to be irrelative to the carrier relaxation.

Since no apparent first and second kind of blocking mechanisms exist in these materials, we infer that a relatively effective up-conversion of acoustic phonons may occur to prolong the relaxation period ${ }^{10}$. Actually, the acoustic phonon up-conversion has been reported recently in the perovskite-like organic-inorganic hybrid multiferroic material ${ }^{50}$. In that material, the existence of organic sub-lattices introduces a series of low-energy 'hybrid phonon' modes, which are defined as the co-vibrations between the organic and inorganic sub-lattices rather than the modes dominated by either kind of sub-lattice alone, according to ref. 50. These low-energy optical-like 'hybrid phonon' cannot be excited efficiently by an external perturbation but by phonon-phonon scatterings instead. Their large thermal coupling rate to acoustic phonons can facilitate the up-conversion of the latter one and prolong the overall relaxation period of hot-phonon populations.

We adopt the phonon classification concept here used in ref. 50 and classify the phonons in organic-inorganic perovskites according to the projected density of states plotted in Fig. 3a-c and also Supplementary Fig. 3a-c. As pointed out before, the co-vibrations between the organic cation and lead/halide ions roughly in $30-100 \mathrm{~cm}^{-1}$ can be called as 'hybrid phonons'. Since initial Fröhlich scattering occurs on the lead-halide framework primarily, these low-energy 'hybrid phonons' are mainly excited by phonon-phonon scattering events and these are very similar to the phonon relaxation process occurring in the multiferroic material discussed in ref. 50. Also, these 'hybrid phonons' showing very little dispersion at around $30 \mathrm{~cm}^{-1}$ have a good overlap with the top of the acoustic branches. This kind of phonon band structure is expected to increase the probability of acoustic phonons scattering to optical states and recycle the vibrational energy, corresponding to a good thermal coupling there. On the basis of this information, we therefore propose that a similar acoustic phonon up-conversion process discussed in ref. 50 may also exist in lead-halide perovskites, especially in the organic-inorganic types. Interestingly, according to the previous comparison, the purely inorganic $\mathrm{CsPbIB}_{2}$ sample with a simpler phonon band structure and reduced phononic DOS do have the fastest carrier relaxation rate in the second cooling stage. 
To further quantify the relaxation rate of the phonon system, we estimate the average LO phonon emission lifetime $\tau_{\text {ave }}$ for the three samples with good quality $\left(\mathrm{FAPbI}_{3}, \mathrm{MAPbBr}_{3}\right.$ and $\mathrm{CsPbBr}_{3}$ ) using equation (1), where $T_{\mathrm{L}}$ is the lattice temperature $^{36}$. Since the elastic scattering between LO phonons and carriers does not dissipate energy irreversibly, the eventual cooling of the carrier-phonon system is caused by the inelastic decay of optical phonons (exciting acoustic phonons). By considering both the emission and reabsorption process of LO modes, the $\tau_{\text {ave }}$ can be taken as an effective cooling lifetime of carriers interacting with a non-equilibrium phonon population $^{35,36,51}$.

$$
-\frac{3 k_{\mathrm{B}}}{2} \frac{\mathrm{d} T_{\mathrm{c}}}{\mathrm{d} t} \approx \frac{\hbar \omega_{\mathrm{LO}}}{\tau_{\mathrm{ave}}}\left[\exp \left(-\frac{\hbar \omega_{\mathrm{LO}}}{k_{\mathrm{B}} T_{\mathrm{c}}}\right)-\exp \left(-\frac{\hbar \omega_{\mathrm{LO}}}{k_{\mathrm{B}} T_{\mathrm{L}}}\right)\right]
$$

We choose the characteristic/effective LO phonon energies for $\mathrm{FAPbI}_{3}$ (ref. 27), $\mathrm{MAPbBr}_{3}$ (refs 52,53), $\mathrm{CsPbBr}_{3}$ (refs 54,55) as $13 \mathrm{meV}, 18 \mathrm{meV}$ and $16 \mathrm{meV}$, respectively, by taking into account both the experimental and theoretical estimations performed before and also the band structure we presented in Fig. 3. These characteristic $\hbar \omega_{\mathrm{LO}}$ are near the top of the optical bands contributed by the lead-halide framework. Complete results for the carrier temperature-dependent LO emission lifetime in each sample are given in Supplementary Fig. 4. All the samples start with a very rapid emission rate $\left.\tau_{\text {ave }}(t)\right|_{t \rightarrow 0} \leq 0.1 \mathrm{ps}$ in the early stage of carrier thermalization without an evident power dependence (Supplementary Fig. 5), since a large non-equilibrium optical phonon population has not yet been achieved in that stage.

In Fig. 4a, we compare the results from the three perovskite samples with good lattice quality. Again, an intermediate excitation intensity $\left(28 \mu \mathrm{J} \mathrm{cm}^{-2}\right.$ for $\mathrm{FAPbI}_{3}, 50 \mu \mathrm{J} \mathrm{cm}{ }^{-2}$ for $\mathrm{MAPbBr}_{3}$ and $\mathrm{CsPbIBr}$ ) is chosen for this comparison to avoid the influence of bleaching saturation in the $\mathrm{MAPbBr}_{3}$ sample under a higher pump fluence. We now focus on the second cooling stage in each sample with a carrier temperature around and below $400 \mathrm{~K}$, where the cooling properties are dominated by the phonon bottleneck effect. We find that $\mathrm{FAPbI}_{3}$ attains a roughly 10 times slower phonon emission rate than that in $\mathrm{CsPbIBr}_{2}$ in this period and around 3 times slower than $\mathrm{MAPbBr}_{3}$, as shown in Fig. 4a. A comparison of the LO emission lifetime between $\mathrm{FAPbI}_{3}$ and $\mathrm{CsPbIBr}$ 2 under the maximum fluences are also provided in Supplementary Fig. 10. A larger increment of $\tau_{\text {ave }}$ is still observed in $\mathrm{FAPbI}_{3}$ leading to an up to 20 times longer emission lifetime than $\mathrm{CsPbIBr}_{2}$ around $T_{\mathrm{c}}=400 \mathrm{~K}$ in that case. Further numerical estimation confirms that this discrepancy does not result from the different LO phonon energies used in the calculation but is dominated by the temperature gradient $-\mathrm{d} T_{\mathrm{c}} / \mathrm{d} t$ among different samples instead. Indeed, the smaller $\mathrm{LO}$ phonon energy used for $\mathrm{FAPbI}_{3}$ decreases the corresponding emission lifetime a little bit according to equation (1). Combined with the previous cooling lifetime comparison, it suggests that a much stronger bottleneck effect exists in $\mathrm{FAPbI}_{3}$ while the pure inorganic counterpart shows the weakest blocking.

The divergence of bottleneck effect observed above could also be explained by different phonon up-conversion efficiencies in these materials, where the proposed up-conversion of acoustic phonon shows a strong correlation to the unique thermal property of lead-halide perovskites. It is expected that the up-conversion of acoustic phonons is accompanied by their rapid and short-range attenuation, which relates microscopically to the thermal conductivity of the material. A strong anharmonic phonon-phonon scattering corresponding to a low thermal conductivity in general can localize acoustic phonons by blocking their propagation and increase the probability of an up-transition. In turn, an efficient up-conversion of acoustic phonon can also impede thermal propagation in the lattice ${ }^{50}$.

In fact, an ultralow thermal conductivity (less than $1 \mathrm{~W} \mathrm{~K}^{-1} \mathrm{~m}^{-1}$ ) has been reported in both of the hybrid type lead-halide perovskites ${ }^{56,57}$ and their $\mathrm{CsPbX}_{3}$ inorganic counterparts ${ }^{58}$. An enhanced anharmonic phonon-phonon scattering resulting from the highly overlapped phonon branches is recognized as the major cause, especially in the hybrid types ${ }^{57}$. The resonant scattering resulting directly from the rotational-like vibrations of organic cation is also contributive ${ }^{56,57}$. Both aspects are consistent with the picture of acoustic phonon up-conversion via the low-energy 'hybrid phonon' modes presented above. In particular, the resonant scattering from the organic cation corresponds to an organic cation-assisted up-conversion route for acoustic phonons, which directly indicates the co-vibrational optical modes can be re-excited by acoustic phonons efficiently in hybrid lead-halide perovskites.
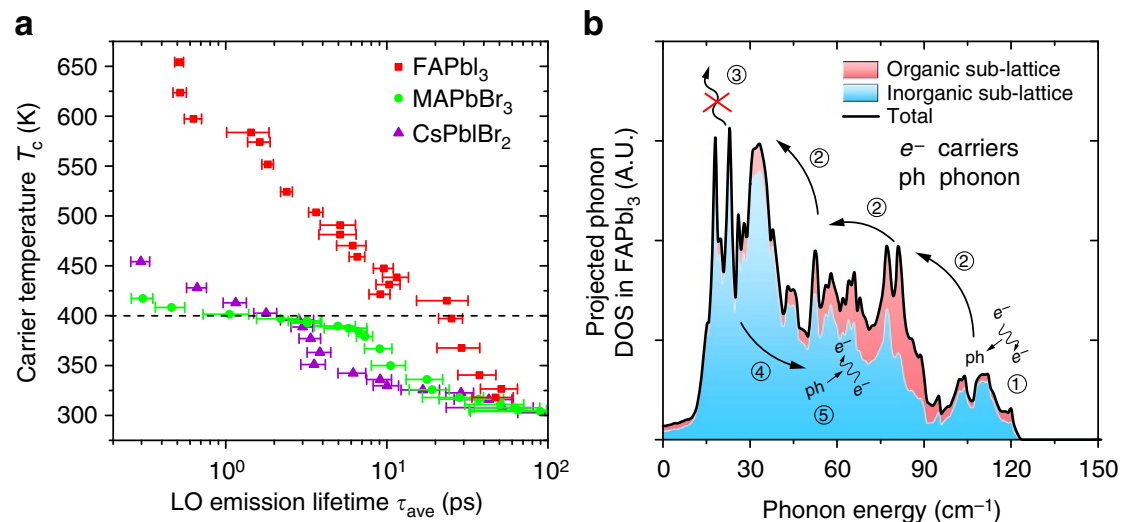

Figure 4 | Phonon dynamics and bottleneck effect in lead-halide perovskites. (a) Carrier temperature-dependent phonon emission lifetime in different leadhalide perovskites with a similar initial carrier concentration of around $2 \times 10^{18} \mathrm{~cm}^{-3}$. The error bars shows the standard error of the average (s.e.m) emission lifetime. An emission lifetime that is about 10 times longer than the other materials is observed in $\mathrm{FAPb}_{3}$ with carrier temperature at and below $400 \mathrm{~K}$; (b) Proposed phonon dynamics in the $\mathrm{FAPbl}_{3}$. The solid black line shows the total phonon DOS, in which the contributions from the inorganic and organic sub-lattices are shown by the blue region on the bottom with the pink region stacked on top, respectively. The labelled phonon dynamic process are: (1) Fröhlich interaction of carriers primarily on the lead-halide framework; (2) relaxation of lead-halide LO phonon, organic sub-lattice can be excited by phonon-phonon scattering; (3) propagation of acoustic phonon is blocked due to anharmonic phonon-phonon scatterings; (4) up-conversion of acoustic phonons; and (5) carrier reheating. 
Now back to the divergence of LO emission lifetime in Fig. 4a. Phonon propagation is blocked in $\mathrm{CsPbIBr}_{2}$ due to its low thermal conductivity, which increases the phonon up-transition probability. However, the lack of the rotatable organic cation and corresponding altered phonon modes leads to a very limit phonon up-conversion efficiency compared with the other two hybrid types. This suggests the indispensable role of the organic cation, or the large amount of low-energy co-vibrational optical modes, in the phonon up-conversion discussed here. The difference between $\mathrm{FAPbI}_{3}$ and $\mathrm{MAPbBr}_{3}$ may result from two aspects. On the one hand, a stronger acoustic phonon localization is expected in the $\mathrm{FAPbI}_{3}$ compared with $\mathrm{MAPbBr}_{3}$. The thermal conductivity of $\mathrm{MAPbBr}_{3}$ has been predicted to be higher than its iodide counterparts due to a higher elastic moduli ${ }^{57}$. Practically, the trigonal phase of $\mathrm{FAPbI}_{3}$ with a lower lattice-symmetry at room temperature is also expected to further limit its thermal conductivity in general ${ }^{59}$. On the other hand, it looks like a better phonon band overlap near the top of acoustic branches is observed in $\mathrm{FAPbI}_{3}$ than those in $\mathrm{MAPbBr}_{3}$ according to Fig. 3 . This could facilitate the re-excitation of optical modes in the former one more efficiently. Overall, the conclusion is that an efficient up-conversion of low-energy phonons discussed here relies on both the blocking of the acoustic phonons propagation and organic cation-assisted phonon up-transition. Both aspects lead to a significant hot-phonon bottleneck in the hybrid leadhalide perovskites, where the hot-phonon relaxation rate in FAPbI3 is observed as at least 10 times slower than that in the inorganic counterpart via LO phonon emission.

\section{Discussion}

In summary, we propose the following carrier-phonon dynamics in hybrid type lead-halide perovskites to explain the observed strong phonon bottleneck effect under a large carrier population. The proposed process is shown in Fig. 4b. (1) First, the Fröhlich interaction occurs predominantly between the hot carriers and inorganic sub-lattice, exciting the high-energy lead-halide LO phonons; (2) the excited LO phonons then decay to acoustic modes. During this stage, the co-vibration between organic and inorganic sub-lattices can also be excited via phonon-phonon scattering; (3) the propagation of acoustic phonons is blocked due to strong anharmonic phonon-phonon scatterings; (4) the up-transition probability of phonons is then increased, especially when organic cations appear in the lattice. The organic cation introduces lots of low-energy co-vibrational optical modes, which overlap well with acoustic branches and facilitate the phonon uptransition; (5) the recycled thermal (vibrational) energy reheats charge carriers and prolongs the overall cooling period of carrierphonon system. It is worth emphasizing that processes (3)-(5) are inefficient in conventional semiconductors whose acoustic phonons can easily propagate away along with the hot carrier cooling. The overall thermal recycling will be more efficient when the phonon density is larger since the probability of phononphonon scattering and up-conversion will further increase, especially if phonon propagation is restricted. This leads to an apparent pumping power-dependent hot-phonon bottleneck effect.

Further considerations about the proposed carrier-phonon dynamics are addressed below.

The mechanism behind the bottleneck effect we have discussed relies on the unique lattice structure and phonon properties of lead-halide perovskites, especially in the organic-inorganic hybrid types. Low-energy optical modes that have a good overlap with acoustic modes need to be introduced to realize a more efficient up-conversion process. This is supposed to be a new and general strategy to achieve long-lived hot carriers in material than opening a large phonon band gap. It is now established that the large band gap is not sufficiently to suppress the decay of hot phonons, especially those via the anti-symmetric Ridley route ${ }^{60}$. Blocking the propagation of acoustic phonons is another aspect to enhance the overall efficiency of up-conversion, since the up-transition probability is usually limited by selection and conservation rules. Materials with a low thermal conductivity are valuable to explore, especially the type with organic-inorganic hybrid compositions.

We also notice that our proposed mechanism has a strong connection to the large polaron effect proposed by Zhu et al. ${ }^{29}$ recently. This theory suggests that the freely rotating organic cation can form a long-range polaron along with charge carriers. The large polaron can screen the hot carriers from scattering with LO phonons. This mechanism only relates to the effective strength of Fröhlich interaction in this material (the first kind of blocking mechanism mentioned above) and dominates in the first cooling stage primarily. Instead, the mechanism we proposed here is attempting to explain the bottleneck effect in the following second cooling stage on a timescale of several hundred picoseconds. These two mechanisms are not in contradiction with each other. In fact, the polaron effect suggests a stronger dynamical coupling between the charge carriers and organic cation directly in lead-halide perovskites, which is usually ignored in the conventional understanding. Our study also indicates the rotatable organic cation plays an important role to the acoustic phonon up-conversion in this kind of material. The direct coupling between carriers and organic cation may further facilitate the thermal recycling in the material, since the vibrational energy transferred onto the organic sub-lattice from low-energy modes could reheat charge carriers more efficiently.

In conclusion, we have investigated the bottleneck effect of carrier-phonon cooling in various lead-halide perovskite thin films with different compositions. By comparing both the carrier cooling lifetime and LO emission rate, we conclude that the hybrid perovskites have a stronger phonon bottleneck effect than the pure inorganic types. The phonon relaxation lifetime in $\mathrm{FAPbI}_{3}$ is estimated to be more than 10 times longer than that in the $\mathrm{CsPbIBr}_{2}$ counterpart under a similar carrier concentration and temperature. The up-conversion of acoustic phonons was proposed as the main factor in the phonon dynamics. In hybrid type perovskites, the low-energy optical phonon modes introduced by the rotatable organic cation show a good band overlap and thermal coupling with the acoustic phonons, allowing an efficient phonon up-conversion. Blocking phonon propagation caused by strong anharmonic scatterings also increases the up-transition probability of low-energy modes. Both aspects mentioned above result in an effective vibrational energy recycling in hybrid lead-halide perovskites and prolong the overall cooling period of the carrier-phonon system. It is valid to note again the poor hot carrier response observed in our $\mathrm{MAPbI}_{3}$ sample despite it having a fairly good performance in conventional solar cells. Special attention to the lattice purity is required in the hot carrier applications. The phonon dynamics discussed here are also expected to motivate new explorations of functional hot carrier materials in future.

\section{Methods}

Sample preparation. $\mathrm{FAPbI}_{3}$. To prepare $1.2 \mathrm{M} \mathrm{HC}\left(\mathrm{NH}_{2}\right)_{2} \mathrm{PbI}_{3}$ solution, $\mathrm{HC}\left(\mathrm{NH}_{2}\right)_{2} \mathrm{I}$ is mixed with $\mathrm{PbI}_{2}$ in dimethylformamide (DMF) at 1:1 mole ratio at room temperature. The solutions with added $\mathrm{HI}$ at specific molar ratio $(0.10 \mathrm{~g}$ of $\mathrm{HI}$ solution $1 \mathrm{ml}$ of perovskite solution) were spread on borosilicate glass and spun at 6,500 r.p.m. for $30 \mathrm{~s}$ using gas-assisted method $^{33}$. The films were dried on a hot plate at $160^{\circ} \mathrm{C}$ for $20 \mathrm{~min}$.

$\mathrm{MAPbBr}_{3}$. All samples were deposited on borosilicate glass substrates. The substrates were cleaned by $2 \%$ Hellmanex detergent, acetone and isopropanol in an ultrasonic bath for $10 \mathrm{~min}$ in each cleaning agent followed by UVO treatment for 
10 min. All $\mathrm{CH}_{3} \mathrm{NH}_{3} \mathrm{PbBr}_{3}$ films were fabricated by vapour-assisted method ${ }^{31}$. First, $\mathrm{PbBr}_{2}$ solution in DMF with a concentration of $1 \mathrm{M}$ was spin-coated on glass substrate at 2,000 r.p.m. for $60 \mathrm{~s}$. After annealing at $70^{\circ} \mathrm{C}$ for $30 \mathrm{~min}$, the film was treated by $\mathrm{CH}_{3} \mathrm{NH}_{3} \mathrm{Br}$ vapour at $175^{\circ} \mathrm{C}$ for $10 \mathrm{~min}$ in a closed glass Petri-dish with $\mathrm{CH}_{3} \mathrm{NH}_{3} \mathrm{Br}$ powder surrounded on a hot plate in a glove box, then rinsed in isopropanol at room temperature, followed by drying in a nitrogen stream.

$M A P b I_{3}$. Unless specified otherwise, all materials were purchased from either Alfa Aesar or Sigma-Aldrich and used as received. $\mathrm{CH}_{3} \mathrm{NH}_{3} \mathrm{I}$ was synthesized by mixing $24 \mathrm{ml} \mathrm{CH} \mathrm{CH}_{3} \mathrm{NH}_{2}$ (33\% in ethanol) and $10 \mathrm{ml} \mathrm{HI} \mathrm{(57 \%} \mathrm{in} \mathrm{water)} \mathrm{in} 100 \mathrm{ml}$ ethanol. After stirring for $2 \mathrm{~h}$, the solvent was removed on a rotary evaporator. The white crystals were dried in a vacuum oven at $60^{\circ} \mathrm{C}$ for $24 \mathrm{~h}$. The soda-lime glass substrates were cleaned and then cut into around $1 \mathrm{~cm}^{2}$. A $25 \mu \mathrm{l} 45 \mathrm{wt} \%$ $\mathrm{CH}_{3} \mathrm{NH}_{3} \mathrm{PbI}_{3}$ DMF solution, prepared from $\mathrm{PbI}_{2}$ and $\mathrm{CH}_{3} \mathrm{NH}_{3} \mathrm{I}$ in a molar ratio of $1: 1$, was spread on it, using a spin-coater. For the conventional spin-coating method, the solution was spun at 6,500 r.p.m. for $30 \mathrm{~s}$, while for the gas-assisted method, a 40 psi dry Argon gas stream was blown over the film during spinning at 6,500 r.p.m. for $2 \mathrm{~s}$ after the spin-coating commenced. The films were then annealed at $100^{\circ} \mathrm{C}$ on a hot plate for $10 \mathrm{~min}$, and then cooled to room temperature on a steel substrate.

$\mathrm{Cs}_{\mathrm{PbIBr}}$. Borosilicate glass was cleaned by sonication in solutions of $2 \%$ Hellmanex in deionized water, acetone and isopropanol for $15 \mathrm{~min}$. After drying, the substrate was treated by UV ozone cleaner for $10 \mathrm{~min}$. A dual source thermal evaporation of the two precursors caesium iodide (CsI) and lead bromide $\left(\mathrm{PbBr}_{2}\right)$ was carried out in a thermal evaporation system (Kurt J. Lesker Mini Spectros) integrated in a glove box. CsI and $\mathrm{PbBr}_{2}$ were loaded in separate crucible heaters and the sample substrates were fixed on a rotatable substrate holder. After the pressure of the evaporator chamber was pumped down to $10-6 \mathrm{mbar}$, CsI and $\mathrm{PbBr}_{2}$ were then heated to the set temperature of $350{ }^{\circ} \mathrm{C}$ and $180^{\circ} \mathrm{C}$, respectively. Once the temperatures were reached, the shutter for each source was opened to commence deposition. The temperature of the substrate holder was kept at $75^{\circ} \mathrm{C}$ during the deposition. The deposition rates of CsI and $\mathrm{PbBr}_{2}$ were set at $0.21 \AA^{-1} \mathrm{~s}^{-1}$ and $0.2 \AA \mathrm{s}^{-1}$, respectively, to achieve a molar ratio of $1: 1$ for the two materials. After the evaporation, the samples were annealed on a hot plate at $250^{\circ} \mathrm{C}$ for $10 \mathrm{~min}$ in the glove box.

Ultrafast transient absorption. Femtosecond pump-probe TA experiments were performed on $\mathrm{MAPbI}_{3}, \mathrm{FAPbI}_{3}, \mathrm{MAPbBr}_{3}$ and $\mathrm{CsPbIBr}$ samples with a TA spectrometer. The laser consisted of a Ti:sapphire mode-locked oscillator that seeded a regenerative amplifier. The output of the amplifier was centred at $800 \mathrm{~nm}$ with a repetition rate of $1 \mathrm{kHz}$ and pulse duration of $100 \mathrm{fs}$, which was then split into pump and probe beamlines. The $400 \mathrm{~nm}$ pump pulses were generated using a $\mathrm{BBO}$ crystal and was attenuated. The probe beam passed through a delay stage and was used to generate a white light continuum. The probe beam was then detected by a polychromatic-CCD. All of the measurements were performed at room temperature. Lattice heating by the pump laser is shown to be a minor effect in our measuments, see Supplementary Note 1 .

Calculation of phonon band structure. The density functional theory calculations were carried out using Quantum Espresso ${ }^{61}$. The Perdew-Ernzerhof-Burke functional ${ }^{62}$ was used to evaluate the ground state properties, electron and phonon properties. The plane-wave energy cutoff was set to $100 \mathrm{Ry}$, while a grid of $6 \times 6 \times 6$ was used for k-point sampling. The tolerance for the electronic system was set to $10^{-12} \mathrm{Ry}$, while that for the phonon calculations was set to $10^{-14} \mathrm{Ry}$. Density functional perturbation theory was used to predict the phonon energies at a $4 \times 4 \times 4$ grid of $\mathbf{Q}$ points for $\mathrm{FAPbI}_{3}, \mathrm{MAPbI}_{3}$ and $\mathrm{CsPbBr}_{3}$. Lattice constants for the calculations are given in Supplementary Table 1. The phonon band structures were then interpolated from this grid using the interatomic force constants. The phonon band structure for $\mathrm{MAPbBr}_{3}$ is extrapolated using the mass approximation approach $^{63}$ with the force constants for $\mathrm{MAPbI}_{3}$.

Data availability. The data that support the findings of this study are available from the corresponding author upon request.

\section{References}

1. Zhou, H. et al. Interface engineering of highly efficient perovskite solar cells. Science 345, 542-546 (2014).

2. Hodes, G. Perovskite-based solar cells. Science 342, 317-318 (2013).

3. Jeon, N. J. et al. Solvent engineering for high-performance inorganic-organic hybrid perovskite solar cells. Nat. Mater. 13, 897-903 (2014).

4. Nie, W. et al. High-efficiency solution-processed perovskite solar cells with millimeter-scale grains. Science 347, 522-525 (2015).

5. Saliba, M. et al. Cesium-containing triple cation perovskite solar cells: Improved stability, reproducibility and high efficiency. Energy Environ. Sci. 9, 1989-1997 (2016).

6. Green, M. A., Ho-Baillie, A. \& Snaith, H. J. The emergence of perovskite solar cells. Nat. Photonics 8, 506-514 (2014).
7. Wen, X. et al. Morphology and carrier extraction study of organic-inorganic metal halide perovskite by one- and two-photon fluorescence microscopy. J. Phys. Chem. Lett. 5, 3849-3853 (2014).

8. Yang, Y. et al. Observation of a hot-phonon bottleneck in lead-iodide perovskites. Nat. Photonics 10, 53-59 (2016).

9. Price, M. B. et al. Hot-carrier cooling and photoinduced refractive index changes in organic-inorganic lead halide perovskites. Nat. Commun. 6, 8420 (2015).

10. Hejda, B. \& Kral, K. Hot-electron cooling and second-generation phonons in polar semiconductors. Phys. Rev. B 47, 15554-15561 (1993).

11. Joshi, R. P. \& Ferry, D. K. Hot-phonon effects and interband relaxation processes in photoexcited GaAs quantum wells. Phys. Rev. B 39, 1180-1187 (1989).

12. Langot, P., Del Fatti, N., Christofilos, D., Tommasi, R. \& Vallee, F. Femtosecond investigation of the hot-phonon effect in GaAs at room temperature. Phys. Rev. B 54, 14487-14493 (1996).

13. Murdin, B., Heiss, W., Langerak, C. \& Lee, S. Direct observation of the LO phonon bottleneck in wide $\mathrm{GaAs} / \mathrm{Al}_{\mathrm{x}} \mathrm{Ga}_{1-\mathrm{x}}$ As quantum wells. Phys. Rev. B 55, 5171-5176 (1997).

14. Pötz, W. Hot-phonon effects in bulk GaAs. Phys. Rev. B 36, 5016-5019 (1987).

15. Kirk, A. P. \& Fischetti, M. V. Fundamental limitations of hot-carrier solar cells. Phys. Rev. B 86, 16520 (2012).

16. Ross, R. T. \& Nozik, A. J. Efficiency of hot carrier solar energy converters. J. Appl. Phys. 53, 3813-3818 (1982).

17. König, D. et al. Hot carrier solar cells: principles, materials and design. Phys. E 42, 2862-2866 (2010).

18. Conibeer, G. et al. Progress on hot carrier cells. Sol. Energy Mater. Sol. Cells 93, 713-719 (2009).

19. Shockley, W. \& Queisser, H. J. Detailed balance limit of efficiency of p-n junction solar cells. J. Appl. Phys. 32, 510-519 (1961).

20. Knight, M. W., Sobhani, H., Nordlander, P. \& Halas, N. J. Photodetection with active optical antennas. Science 332, 702-704 (2011).

21. Ni, X. et al. InGaN staircase electron injector for reduction of electron overflow in InGaN light emitting diodes. Appl. Phys. Lett. 97, 31110 (2010).

22. Mukherjee, S. et al. Hot electrons do the impossible: plasmon-induced dissociation of $\mathrm{H}_{2}$ on Au. Nano Lett. 13, 240-247 (2013).

23. Clavero, C. Plasmon-induced hot-electron generation at nanoparticle/metaloxide interfaces for photovoltaic and photocatalytic devices. Nat. Photonics $\mathbf{8}$, 95-103 (2014).

24. Gu, Z. et al. Two-photon pumped $\mathrm{CH}_{3} \mathrm{NH}_{3} \mathrm{PbBr}_{3}$ perovskite microwire lasers. Adv. Opt. Mater. 4, 472-479 (2016).

25. Yakunin, S. et al. Low-threshold amplified spontaneous emission and lasing from colloidal nanocrystals of caesium lead halide perovskites. Nat. Commun. 6, 8056 (2015).

26. Xing, G. et al. Low-temperature solution-processed wavelength-tunable perovskites for lasing. Nat. Mater. 13, 476-480 (2014).

27. Wright, A. D. et al. Electron-phonon coupling in hybrid lead halide perovskites. Nat. Commun. 7, 11755 (2016).

28. Mueller, M. L., Yan, X., Dragnea, B. \& Li, L. Slow hot-carrier relaxation in colloidal graphene quantum dots. Nano Lett. 11, 56-60 (2010).

29. Zhu, X. Y. \& Podzorov, V. Charge carriers in hybrid organic-inorganic lead halide perovskites might be protected as large polarons. J. Phys. Chem. Lett. 6, 4758-4761 (2015).

30. Huang, F. et al. Gas-assisted preparation of lead iodide perovskite films consisting of a monolayer of single crystalline grains for high efficiency planar solar cells. Nano Energy 10, 10-18 (2014).

31. Sheng, R. et al. Methylammonium lead bromide perovskite-based solar cells by vapour-assisted deposition. J. Phys. Chem. C 119, 3545-3549 (2015).

32. Ma, Q., Huang, S., Wen, X., Green, M. A. \& Ho-Baillie, A. W. Y. Hole transport layer free inorganic $\mathrm{CsPbIBr}_{2}$ perovskite solar cell by dual source thermal evaporation. Adv. Energy Mater. 6, 1502202 (2016).

33. Kim, J. et al. Nucleation and growth control of $\mathrm{HC}\left(\mathrm{NH}_{2}\right)_{2} \mathrm{PbI}_{3}$ for planar perovskite solar cell. J. Phys. Chem. C 120, 11262-11267 (2016).

34. Ryan, J. F. et al. Time-resolved photoluminescence of two-dimensional hot carriers in GaAs-AlGaAs heterostructures. Phys. Rev. Lett. 53, 1841-1844 (1984).

35. von der Linde, D. \& Lambrich, R. Direct measurement of hot-electron relaxation by picosecond spectroscopy. Phys. Rev. Lett. 42, 1090-1093 (1979).

36. Zanato, D., Balkan, N., Ridley, B. K., Hill, G. \& Schaff, W. J. Hot electron cooling rates via the emission of LO-phonons in InN. Semicond. Sci. Technol. 19, 1024-1028 (2004).

37. Kawai, H., Giorgi, G., Marini, A. \& Yamashita, K. The mechanism of slow hot-hole cooling in lead-iodide perovskite: first-principles calculation on carrier lifetime from electron-phonon interaction. Nano Lett. 15, 3103-3108 (2015).

38. Chang, Y. H., Park, C. H. \& Matsuishi, K. First-principles study of the structural and the electronic properties of the lead-halide-based inorganicorganic perovskites $\left(\mathrm{CH}_{3} \mathrm{NH}_{3}\right) \mathrm{PbX}_{3}$ and $\mathrm{CsPb}_{3}(\mathrm{X}=\mathrm{Cl}, \mathrm{Br}, \mathrm{I})$. J. Korean Phys. Soc. 44, 889-893 (2004). 
39. Wen, X. et al. Defect trapping states and charge carrier recombination in organic-inorganic halide. J. Mater. Chem. C 4, 793-800 (2015).

40. Zhang, Z. et al. Effect of halide treatments on PbSe quantum dot thin films: Stability, hot carrier lifetime, and application to photovoltaics. J. Phys. Chem. C 119, 24149-24155 (2015).

41. Beard, M. C. Multiple exciton generation in semiconductor quantum dots. J. Phys. Chem. Lett. 2, 1282-1288 (2011).

42. Yang, Y. et al. Comparison of recombination dynamics in $\mathrm{CH}_{3} \mathrm{NH}_{3} \mathrm{PbBr}_{3}$ and $\mathrm{CH}_{3} \mathrm{NH}_{3} \mathrm{PbI}_{3}$ perovskite films: influence of exciton binding energy. J. Phys. Chem. Lett. 6, 4688-4692 (2015).

43. Patterson, R. et al. Phonon lifetimes in model quantum dot superlattice systems with applications to the hot carrier solar cell. Sol. Energy Mater. Sol. Cells 94, 1931-1935 (2010).

44. Conibeer, G. J., König, D., Green, M. A. \& Guillemoles, J. F. Slowing of carrier cooling in hot carrier solar cells. Thin Solid Films 516, 6948-6953 (2008).

45. Brivio, F., Butler, K. T., Walsh, A. \& van Schilfgaarde, M. Relativistic quasiparticle self-consistent electronic structure of hybrid halide perovskite photovoltaic absorbers. Phys. Rev. B 89, 155204 (2014).

46. Frost, J. M. et al. Atomistic origins of high-performance in hybrid halide perovskite solar cells. Nano Lett. 14, 2584-2590 (2014).

47. Mosconi, E., Amat, A., Nazeeruddin, M. K., Grätzel, M. \& De Angelis, F. First-principles modeling of mixed halide organometal perovskites for photovoltaic applications. J. Phys. Chem. C 117, 13902-13913 (2013).

48. Motta, C. et al. Revealing the role of organic cations in hybrid halide perovskite $\mathrm{CH}_{3} \mathrm{NH}_{3} \mathrm{PbI}_{3}$. Nat. Commun. 6, 7026 (2015).

49. Quarti, C. et al. The raman spectrum of the $\mathrm{CH}_{3} \mathrm{NH}_{3} \mathrm{PbI}_{3}$ hybrid perovskite: Interplay of theory and experiment. J. Phys. Chem. Lett. 5, 279-284 (2014).

50. Caretta, A. et al. Measurement of the acoustic-to-optical phonon coupling in multicomponent systems. Phys. Rev. B 91, 1-6 (2015).

51. Ridley, B. K. Hot phonons in high-field transport. Semicond. Sci. Technol. 4, 1142-1150 (1999).

52. Ledinský, M. et al. Raman spectroscopy of organic-inorganic halide perovskites. J. Phys. Chem. Lett. 6, 401-406 (2015).

53. Kunugita, H. et al. Excitonic feature in hybrid perovskite $\mathrm{CH}_{3} \mathrm{NH}_{3} \mathrm{PbBr}_{3}$ single crystals. Chem. Lett. 44, 852-854 (2015).

54. Yunakova, O. N., Miloslavskii, V. K. \& Kovalenko, E. N. Exciton absorption spectrum of thin $\mathrm{CsPbI}_{3}$ and $\mathrm{Cs}_{4} \mathrm{PbI}_{6}$ films. Opt. Spectrosc. 112, 91-96 (2012).

55. Sebastian, M. et al. Excitonic emissions and above-band-gap luminescence in the single-crystal perovskite semiconductors $\mathrm{CsPbBr}_{3}$ and $\mathrm{CsPbCl}_{3}$. Phys. Rev. B 92, 235210 (2015)

56. Pisoni, A., Baris, O. S., Spina, M. \& Gaa, R. Ultra-low thermal conductivity in organic-inorganic hybrid perovskite $\mathrm{CH}_{3} \mathrm{NH}_{3} \mathrm{PbI}_{3}$. J. Phys. Chem. Lett. 5, 2488-2492 (2014).

57. Wang, M. \& Lin, S. Anisotropic and ultralow phonon thermal transport in organic-inorganic hybrid perovskites: atomistic insights into solar cell thermal management and thermoelectric energy conversion efficiency. Adv. Funct. Mater. 26, 5297-5306 (2016).

58. Kubičár, L., Boháč, V. \& Nitsch, K. Thermophysical properties of the $\mathrm{CsPbCl}_{3}$ single crystal using pulse transient method. Int. J. Thermophys. 21, 571-583 (2000).

59. Qian, X., Gu, X. \& Yang, R. Lattice thermal conductivity of organic-inorganic hybrid perovskite $\mathrm{CH}_{3} \mathrm{NH}_{3} \mathrm{PbI}_{3}$. Appl. Phys. Lett. 63902, 3-8 (2015).
60. Xia, H. et al. Numerical calculation of optical phonon decay rate in InN/GaN MQW. IOP Conf. Ser. Mater. Sci. Eng. 68, 12009 (2014).

61. Giannozzi, P. et al. QUANTUM ESPRESSO: a modular and open-source software project for quantum simulations of materials. J. Phys. Condens. Matter. 21, 395502 (2009).

62. Perdew, J. P., Ernzerhof, M. \& Burke, K. Rationale for mixing exact exchange with density functional approximations. J. Chem. Phys. 105, 9982 (1996).

63. Giannozzi, P., de Gironcoli, S., Pavone, P. \& Baroni, S. Ab initio calculation of phonon dispersions in semiconductors. Phys. Rev. B 43, 7231-7242 (1991).

\section{Acknowledgements}

The Australian Centre for Advanced Photovoltaics (ACAP) encompasses the Australian-based activities of the Australia U.S. Institute for Advanced Photovoltaics (AUSIAPV) and is supported by the Australian Government through the Australian Renewable Energy Agency (ARENA). Responsibility for the views, information or advice expressed herein is not accepted by the Australian Government. J.Y. thanks the China Scholarship Council (CSC) for scholarship support (No 201306070023).

\section{Author contributions}

X.W. conceived the project; J.Y. analysed experimental results and wrote manuscript with assistance from X.W., H.X. and R.P.; J.Y. and H.X. performed theoretical calculation; X.W. performed experiments with assistance from P.T., T.H. and T.W.K.; R.S., Q.M., J.K. and F.H. fabricated samples; all authors contributed to the discussion of results and commented on the manuscript.

\section{Additional information}

Supplementary Information accompanies this paper at http://www.nature.com/ naturecommunications

Competing financial interests: The authors declare no competing financial interests.

Reprints and permission information is available online at http://npg.nature.com/ reprintsandpermissions/

How to cite this article: Yang, J. et al. Acoustic-optical phonon up-conversion and hot-phonon bottleneck in lead-halide perovskites. Nat. Commun. 8, 14120 doi: $10.1038 /$ ncomms14120 (2017).

Publisher's note: Springer Nature remains neutral with regard to jurisdictional claims in published maps and institutional affiliations.

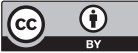

This work is licensed under a Creative Commons Attribution 4.0 International License. The images or other third party material in this article are included in the article's Creative Commons license, unless indicated otherwise in the credit line; if the material is not included under the Creative Commons license, users will need to obtain permission from the license holder to reproduce the material. To view a copy of this license, visit http://creativecommons.org/licenses/by/4.0/

(C) The Author(s) 2017 